function and related genetic variation could be used to predict the efficacy and safety of drugs that raise concentrations of HDL cholesterol.

The author thanks the late Jarmo Pikkarainen and Georg Alfthan, National Public Health Institute of Finland, for measurements of $\gamma$-glutamyltransferase and Kari Seppänen for lipoprotein analyses.

Contributor: JTS initiated the Kuopio ischaemic heart disease (KIHD) risk factor study, analysed the data, wrote the paper, and is the guarantor.

Funding: The KIHD study was funded by research grants from the National Institutes of Health (grant HL 44199 to professor George A Kaplan) and from the Academy of Finland (grants 41471, 1041086, and 2041022 to J T Salonen).

Competing interests: JTS is the inventor in a related patent application (WO 03/052129).
1 Salonen JT, Salonen R, Seppänen K, Rauramaa R, Tuomilehto J. High density lipoprotein, $\mathrm{HDL}_{2}$ and $\mathrm{HDL}_{3}$ subfractions and the risk of acute myocardial infarction: a prospective population study in Eastern Finnish men. Circulation 1991;84:129-39.

2 Salonen JT, Ylä-Herttuala S, Yamamoto R, Butler S, Korpela H, Salonen $\mathrm{R}$, et al. Autoantibody against oxidised LDL and progression of carotid atherosclerosis. Lancet 1992;339:883-7.

3 Perova NV, Oganov RG, Williams DH, Irving SH, Abernathy JR, Deev AD, et al. Association of high-density-lipoprotein cholesterol with mortality and other risk factors for major chronic noncommunicable diseases in samples of US and Russian men. Ann Epidemiol 1995;5:179-85.

4 Liinamaa MJ, Hannuksela ML, Kesäniemi YA, Savolainen MJ. Altered transfer of cholesterol esters and phospholipids in plasma from alcohol abusers. Arterioscler Thromb Vasc Biol 1997;17:2940-7.

5 Committee of Enzymes of the Scandinavian Society for Clinical Chemistry and Clinical Physiology. Recommended method for the determiantion of gamma-glutamyl transferase in blood. Scand J Clin Lab Invest 1976;36:119-25.

(Accepted 4July 2003)

\title{
Number of published systematic reviews and global burden of disease: database analysis
}

\author{
George H Swingler, Jimmy Volmink, John P A Ioannidis
}

Systematic reviews are key to implementing evidence based medicine. ${ }^{1}$ We wondered if the reviews done to date are related to the burden of disease from various conditions. Ideally, evidence should be prioritised for diseases with the greatest global impact.

\section{Methods and results}

We estimated Spearman correlations between the number of systematic reviews in two important databases (the Cochrane database of systematic reviews (CDSR) and the database of abstracts of reviews of effects (DARE)) and the burden of disease (globally and in established market economies) across disease categories. We also estimated the burden of disease for each available review measured in disability adjusted life years (DALYs). ${ }^{23}$ We used 1990 estimates of burden of disease because studies included in systematic reviews would have responded to recent past health needs. Results with estimates from 2000 were similar.

We categorised tar geted diseases in 923 reviews from the CDSR and 1899 reviews from the DARE in issue 4, 2000, of the Cochrane Library using 20 categories of the global burden of disease taxonomy. ${ }^{3}$ We excluded unclassifiable topics (health systems, pain or anaesthesia, general operative techniques, and smoking cessation). To avoid small contributors to burden of disease, a separate analysis retained only the top 10 groups of disease accounting for $>90 \%$ of the global burden of disease. Reviews in the DARE came from high profile general medical journals (173), other general journals (77), specialist journals (1532), or other reports (117). Two independent investigators did categorisations and resolved disagreements by discussion.

We looked for correlation between the number of systematic reviews and the burden of disease. Given the small number of categories, modest differences in estimated correlations between databases and subgroups should not be attributed formal statistical significance.
We categorised 866 reviews from the CDSR and 1639 reviews from the DARE (898 and 1729 disease group entries). Coverage was similar across databases except the CDSR covered maternal and perinatal conditions better. Across disease groups, global DALYs for each review varied between 0.2-33.0 million in the CDSR and 0.1-5.5 million in the DARE. Among the top 10 disease groups, nutritional deficiencies, injuries, respiratory infections, and infectious diseases were most neglected ( $>2$ million global DALYs for each available review in either database).

Burden of disease was modestly correlated with the number of systematic reviews in the CDSR (global $\mathrm{r}=0.54, \quad \mathrm{P}=0.014$; established market economies $\mathrm{r}=0.46, \quad \mathrm{P}=0.041$ ), the DARE (global $\mathrm{r}=0.65$, $\mathrm{P}=0.002$; established market economies $\mathrm{r}=0.76$, $\mathrm{P}<0.001)$ and in subgroups of the DARE.

For the top 10 disease groups, correlations between the number of systematic reviews and the global burden of disease remained unchanged in CDSR $(\mathrm{r}=0.52, \mathrm{P}=0.13)$, but decreased in DARE $(\mathrm{r}=0.42$, $\mathrm{P}=0.23$ ). The burden of disease in established market economies correlated modestly with the number of reviews in the CDSR $(\mathrm{r}=0.56 ; \mathrm{P}=0.09)$; correlations in the DARE were high (overall $\mathrm{r}=0.87, \mathrm{P}<0.001$, range 0.63-0.94 across subgroups of reviews).

The number of reviews in the DARE seemed less responsive to global burden of disease than to the burden in established market economies, but the difference was not significant. The CDSR did not show this (figure).

\section{Comment}

The number of published systematic reviews is still relatively small and unevenly covers different diseases and aspects of health care. Often, millions of DALYs correspond to each available systematic review. Of course, new systematic reviews are continuously
School of Child and Adolescent Health, Red Cross

Children's Hospital, University of Cape Town, 7700

Rondebosch, South Africa

George H Swingler associate professor

Primary Health Care, Faculty of Health Sciences, University of Cape Town, Cape Town Jimmy Volmink professor

Clinical Trials and Evidence-Based Medicine Unit, Department of Hygiene and Epidemiology, University of Ioannina School of Medicine, Ioannina 45110, Greece

John P A Ioannidis chairman

Correspondence to: $\mathrm{J}$ P A Ioannidis jioannid@cc.uoi.gr

BMJ 2003;327:1083-4

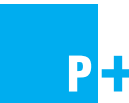

The disease categories in the figure are in order on bmi.com 

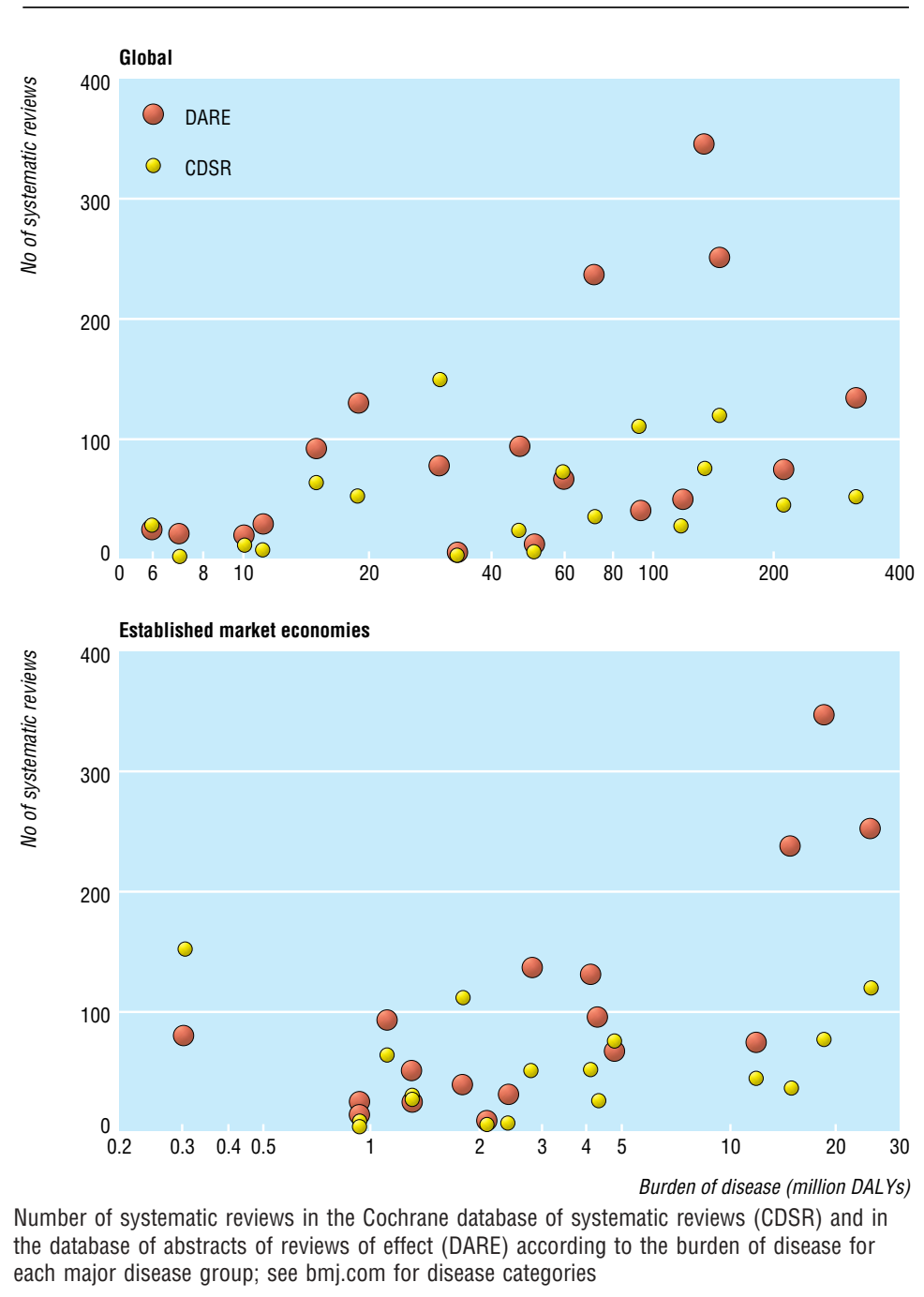

produced: there are currently 5877 reviews in the Cochrane Library (issue 3, 2004, including 1754 in
CDSR and 4123 in DARE), but the overall picture is probably the same. Differences between the CDSR and the DARE may be because the CDSR is an electronic database without space limitations. Conversely, the content in the DARE depends more on selection forces related to the subject matter with preference for health problems in established market economies. In peer reviewed journals (and the database of abstracts of reviews of effect (DARE)), systematic reviews follow the priorities of established market economies rather than global priorities.

Measuring research and the emphasis of research is difficult. ${ }^{4}$ The availability of synthesised knowledge is a product of the amount of primary research. For most healthcare practitioners, synthesised knowledge is more relevant than primary research. Also, doing systematic reviews is important even if evidence is lacking. ${ }^{5}$ Systematic reviews fathom the existing uncertainty and help build an agenda for future research.

Contributors: JPAI and GHS designed the study. GHS coordinated the study. JPAI did the analysis. All authors extracted data, interpreted findings, and wrote the report. JPAI is guarantor.

Funding: No additional funding.

Competing interests: All three authors have been involved in the Cochrane Collaboration.

Ethical approval: Not needed.

1 Mulrow CD, Cook DJ, Davidoff F. Systematic reviews: critical links in the great chain of evidence. Ann Intern Med 1997;126:389-91.

2 Isaakidis P, Swingler GH, Pienaar E, Volmink J, Ioannidis JPA. Burden of disease and randomized evidence in sub-Saharan Africa. BMJ 2002;324:702-4.

Murray CJL, Lopez AD. The global burden of disease, a comprehensive assessment of mortality and disability from diseases, injuries, and risk factors in 1990 and projected to 2020. Harvard: Harvard University Press, 1996.

4 Gross CP, Anderson GF, Powe NR. The relation between funding by the National Institutes of Health and the burden of disease. $N$ Engl J Med 1999;340:1881-7.

5 Alderson P, Roberts I. Should journals publish systematic reviews that find no evidence to guide practice? Examples from injury research. $B M J$ 2000;320:376-7.

(Accepted 4 August 2003)

\section{A memorable patient}

\section{Don't place all your trust in machines}

While attending a post-take $\mathrm{x}$ ray meeting as an orthopaedic senior house officer, I presented my sixth femoral neck fracture of the night. "Mrs X is a an elderly lady from a residential home, with left ventricular failure and advanced dementia. She was found on the floor in her room in the early hours of this morning, unable to weight bear. Plain radiographs show she has sustained an undisplaced subcapital fractured neck of femur on the right," I said as I flashed the radiograph up onto the screens.

My audience was quick to point out that the radiograph clearly showed a left sided injury, with the right side being normal. My consultant asked the on-call registrar, who couldn't remember the side, but the radiographer present in the meeting was eager to assert that, with the advent of digital imaging, "the laterality marker is placed on the radiograph by the computer so it must be correct, it must be the SHO who has made the mistake." As I expect any other tired senior house officer would have done, I apologised and made a mental note to change the operating list.

When we arrived at my patient on the post-take ward round the team launched into another discussion of how important it was to identify the correct side of injury, since one would be a long way into a hemi-arthroplasty before finding that a mistake had been made. Because of her dementia, the patient was unable to contribute to the discussion. The registrar then drew back the sheets to show the large black arrow she had drawn the night before, and the grimace on the patient's face when the leg was rolled in the bed. As expected from an undisplaced fracture, there was no deformity. Suggestions about how one should avoid making such mistakes were volunteered. Thoroughly disheartened, I kept quiet.

Fortunately, the consultant took the time to examine the "good side." On drawing back the other side of the sheets, he found the large black arrow that I had drawn the night before. On his moving the right leg, the patient displayed a great deal more discomfort, and it was obvious the fracture was on the right!

Needless to say, I am now careful to be a little more trusting of my memory and little less trusting of machines.

C A Willis-Owen senior house officer, paediatric surgery, John Radcliffe Hospital, Oxford 